\title{
Effects of Methane Fermentation on Spectral Properties of Fulvic Acid Extracted from Peat through Liquid Acid Precipitation
}

\author{
Li-Tong Ma $\mathbb{D}^{1,2}$ Ya-Nan Lu, ${ }^{1}$ and Ya-Xiong Wang ${ }^{1,3}$ \\ ${ }^{1}$ School of Chemistry and Chemical Engineering, Inner Mongolia University of Science and Technology, Baotou 014010, \\ Inner Mongolia Autonomous Region, China \\ ${ }^{2}$ Inner Mongolia Engineering Research Center of Comprehensive Utilization of Bio-Coal Chemical Industry, Baotou 014010, \\ Inner Mongolia Autonomous Region, China \\ ${ }^{3}$ Inner Mongolia Key Laboratory of Coal Chemical Industry Comprehensive Utilization, Baotou 014010, \\ Inner Mongolia Autonomous Region, China
}

Correspondence should be addressed to Li-Tong Ma; mlt0916@126.com

Received 2 December 2019; Revised 23 March 2020; Accepted 13 April 2020; Published 22 May 2020

Academic Editor: Mohamed Azaroual

Copyright (C) 2020 Li-Tong Ma et al. This is an open access article distributed under the Creative Commons Attribution License, which permits unrestricted use, distribution, and reproduction in any medium, provided the original work is properly cited.

The effects of acid precipitation with different liquid acids on spectral properties of fulvic acid extracted from peat was studied to select the most appropriate liquid acid for the acid precipitation step of the alkali solution acid precipitation method, and the new process mechanism of peat utilization by coupling methane fermentation and extraction of fulvic acid was analyzed. The alkali solution acid precipitation method was adopted to extract fulvic acid from the methane-fermented group and methane-unfermented group. Different liquid acids were used to conduct acid precipitation during the extraction. And then, the characterizations of fulvic acid samples were conducted through Fourier infrared spectroscopy, UV-Vis spectroscopy, and fluorescence spectroscopy. The yield and content of fulvic acid decreased significantly after methane fermentation. During the methane fermentation process, some fulvic acid was consumed and utilized by microorganisms to promote the methane fermentation process, resulting both in the decrease of methyl, hydroxyl, and ether bonds and in the increase of methylene, carbonyl, conjugated double bond, benzene rings, and other groups. The $E_{4} / E_{6}$ ratio shows that the $E_{4} / E_{6}$ ratio of fulvic acid was decreased after methane fermentation, and the fermentation consumed the functional group with simpler structure such as aliphatic chain hydrocarbon of fulvic acid while the structure with a higher degree of aromatization and conjugate cannot be consumed. When conducting acid precipitation with different liquid acids, the yield of fulvic acid was the highest through acid precipitation with phosphoric acid. The fulvic acid obtained through acid precipitation with nitric acid has a higher content, more benzene rings, and the highest degree of aromatization.

\section{Introduction}

There are abundant coal resources within Inner Mongolia, including peat, anthracite, and lignite. The main components of peat include organic matter (53\% on average) and humic acid (36\% on average) [1]. It has more pores, huge internal surface area, and strong ability to adsorb water, which are commonly used in flower gardening [2], crop planting [3], and soil improvement [4] in Germany, Finland, Canada, Sweden, and China. However, it is a waste to use peat only for horticultural substrate and organic fertilizer. Peat should be positioned as a raw material to improve its economic value.
As the main chemical component of peat, fulvic acid (FA) is a macromolecular substance with benzene rings as the basic unit. There are several active functional groups such as carboxyl, hydroxyl, and methoxy distributed on the benzene rings and side chains [5]. Fulvic acid, soluble in alkali, acid, and water, is the best core component of soil humus [6] and also the best plant growth regulator [7].

The alkali solution acid precipitation is the common extraction method of fulvic acid. Trofimova et al. [8] studied the humic acid extracted from lowland peat and upland peat by the alkali solution acid precipitation method. The results showed that the humic acid extracted from the lowland peat 
TABLe 1: Fulvic acid in different experiment groups.

\begin{tabular}{lccc}
\hline Test group & Peat $(\mathrm{g})$ & Methane fermentation & Acid precipitation \\
\hline FMS & 40 & $\sqrt{ }$ & Sulfuric acid \\
FMN & 40 & $\sqrt{ }$ & Nitric acid \\
FMH & 40 & $\sqrt{ }$ & Hydrochloric acid \\
FMP & 40 & $\sqrt{ }$ & Phosphoric acid \\
FS & 40 & & Sulfuric acid \\
FN & 40 & & Nitric acid \\
FH & 40 & & Hydrochloric acid \\
FP & 40 & & Phosphoric acid \\
\hline
\end{tabular}

types contained large amounts of aromatic carbon, phenolic and alcohol groups, carbohydrate residues, and ethers; the humic acid of the upland peat types owned high content of carbonyl, carboxyl, and ester groups. Yuanping et al. [9] explored the optimal process for extracting biochemical humic acid from the fermented furfural residue by the alkali solution acid precipitation method. It was found that solid biochemical humic acid with humic acid content of $76 \%$ and yield of $49 \%$ was obtained under the conditions as follows: the solid-liquid ratio was $1: 7$ during the alkali solution step, the $\mathrm{KOH}$ concentration was $8 \%$, the extraction time was 1 hour, the extraction temperature was $70^{\circ} \mathrm{C}$, and the $\mathrm{pH}$ was 2.5 during the acid precipitation step.

In terms of the current research report on the extraction of fulvic acid from peat, they paid more attention to the method of extracting fulvic acid, so this ignored the full usage of peat. And, Yuanping et al. [9] only found the best $\mathrm{pH}$ was adjusted by hydrochloric acid; they did not study the effect of extracting fulvic acid by the alkali solution acid precipitation method with different liquid acids. With herbaceous peat as the research object in this experiment, we studied the effects of methane fermentation on spectral characteristic change of fulvic acid extracted from peat by the alkali solution acid precipitation method and analyzed the mechanism of coupling peat methane fermentation and extracting fulvic acid. At the same time, we studied the effects of fulvic acid extracted from peat by different liquid acid precipitation to find the most appropriate liquid acid for precipitation.

We expected to realize the superposition of peat conversion benefits and improve the overall economic benefits of peat resource through the staged conversion of chemical components of peat including organic matter and humic acid, laying the foundation for the industrial extraction of fulvic acid from peat.

\section{Materials and Methods}

2.1. Instruments and Reagents. Herbaceous peat was provided by Jilin Jixiang Co., Ltd.; activated sludge was obtained from the sewage treatment plant located in a southern suburb of Baotou; chromatographic pure fulvic acid (FC) was provided by the Fusheng Industry. The automatic methane potential test system (AMPTS II) was purchased from the Bioprocess Company of Sweden; the Fourier-transform infrared (FTIR) spectrometer (RX1) was purchased from Germany Bruker; the UV-visible

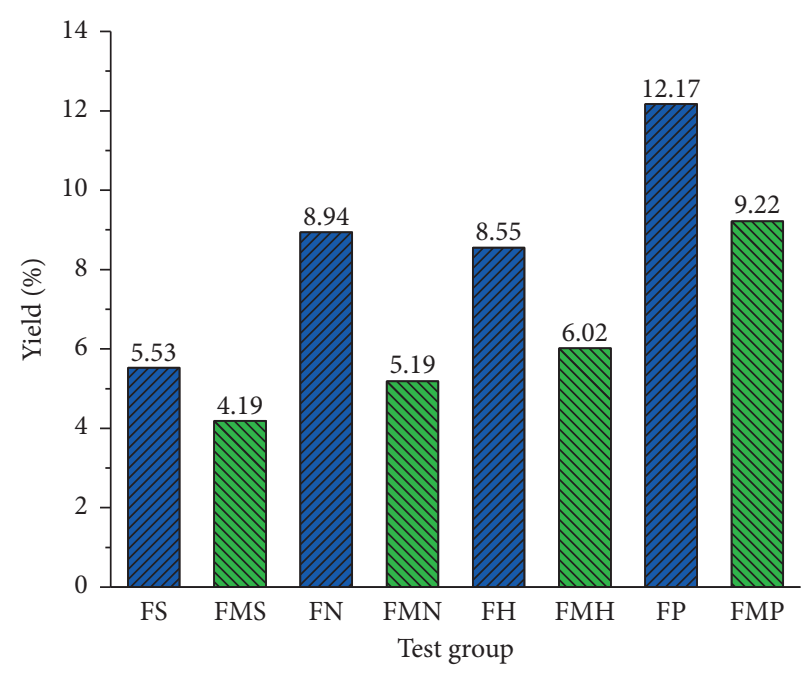

Figure 1: Yield of fulvic acid.

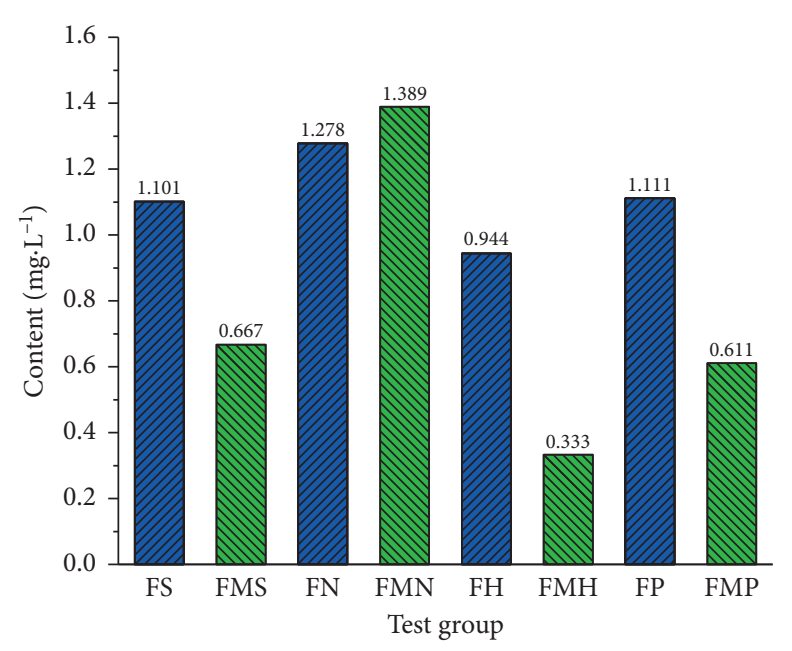

Figure 2: Content of fulvic acid.

near-infrared (UV-VIS) spectrophotometer (CAYR 5 000) was purchased from Varian; the fluorescence luminescence spectrometer (LS55) was purchased from PerkinElmer.

\subsection{Experimental Method}

2.2.1. Methane Fermentation Group. The automatic methane potential test system (AMPTS II) of Bioprocess Company of Sweden was adopted as the fermentation experiment equipment. The $200 \mathrm{~mL}$ activated sludge and $40 \mathrm{~g}$ peat sieved into a size of 100 mesh (Table 1 below) were added in a fermentation system, then the initial $\mathrm{pH}$ was adjusted to 7.0, and the water was added until the volume of the total fermentation system was $400 \mathrm{~mL}$. It was then flushed with pure nitrogen for 120 seconds to provide an anaerobic condition. Finally, the fermentation temperature was adjusted to $50^{\circ} \mathrm{C}$. In the reaction process, the stirring was controlled automatically by the motor, and the stirring interval was 6 hours for each stirring of 


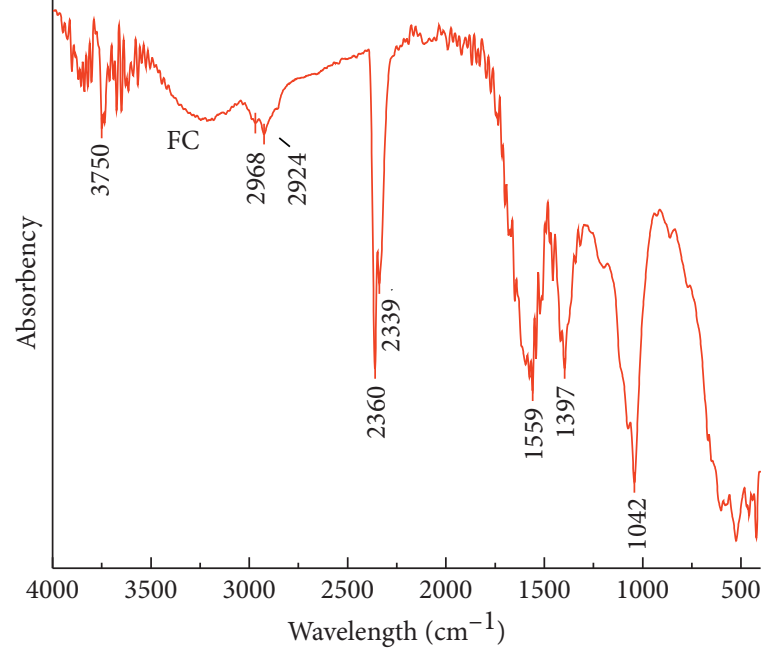

(a)

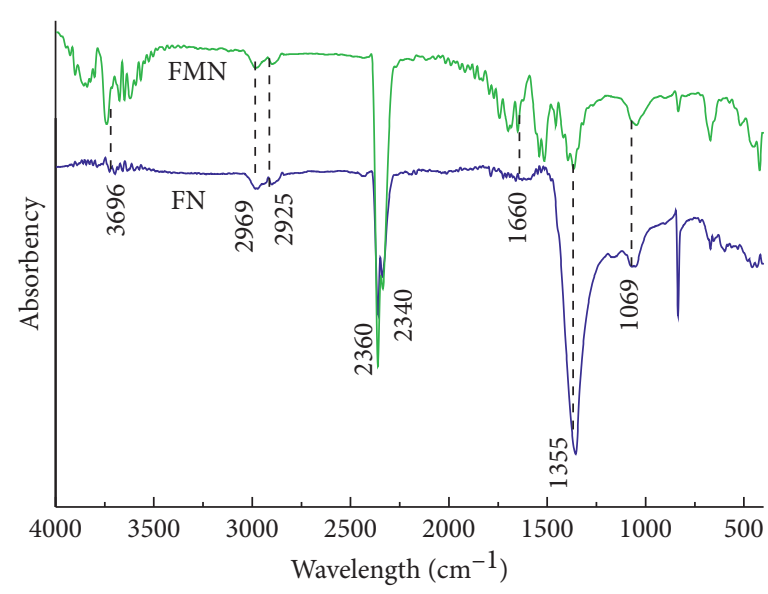

(c)

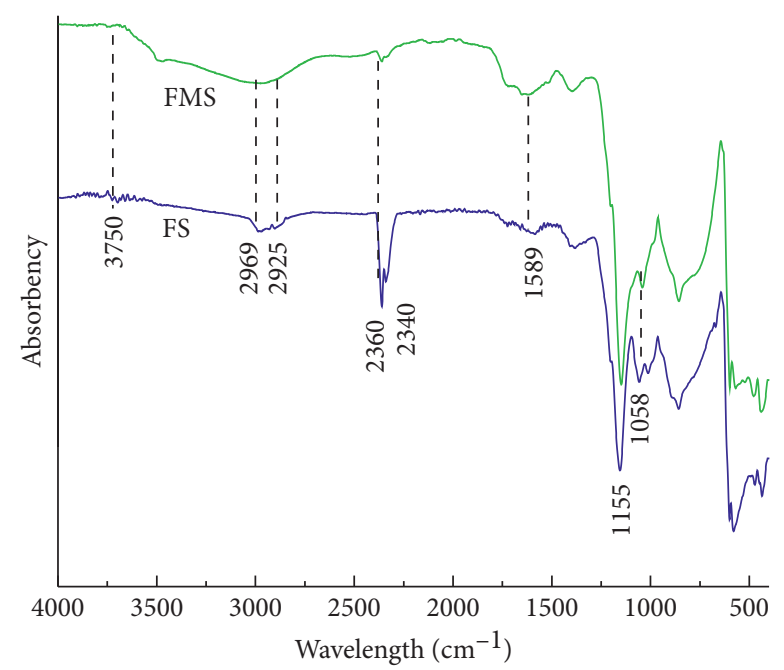

(b)

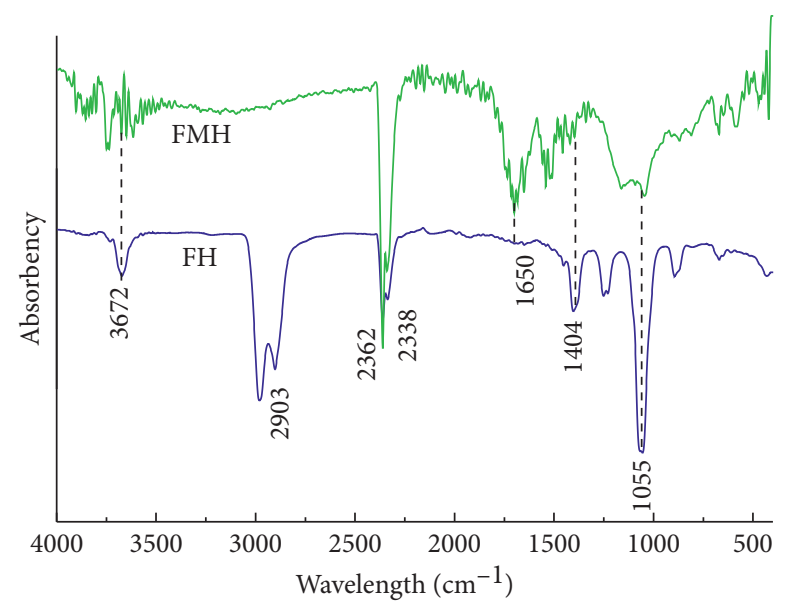

(d)

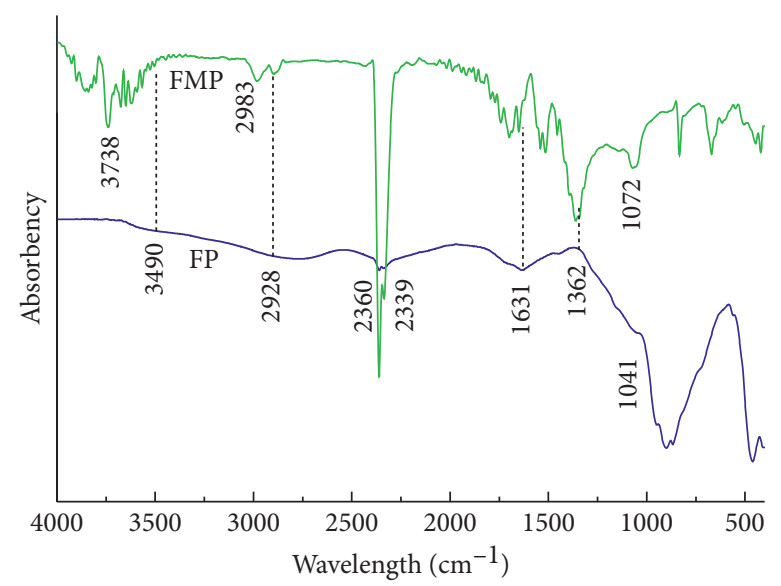

(e)

FIGURE 3: (a) FTIR of fulvic acid. FTIR of fulvic acid by (b) sulfuric acid precipitation, (c) nitric acid precipitation, (d) hydrochloric acid precipitation, and (e) phosphoric acid precipitation.

$10 \mathrm{~min}$. The daily methane production and total methane production were recorded by the instrument automatically. Three parallel experiments were carried out in each group, and the average value was taken. After the completion of fermentation, the residue was dried to extract fulvic acid for testing. In the unfermented group, fulvic acid was extracted from herbaceous peat directly for testing. 
TABLE 2: Infrared peak position comparison.

\begin{tabular}{lcc}
\hline Peak position & Functional group & Absorption type \\
\hline $3700 \sim 3200 \mathrm{~cm}^{-1}$ & Hydroxyl $(-\mathrm{OH})$ & Stretching vibration \\
$2960 \pm 10 \mathrm{~cm}^{-1}$ & Methyl $\left(-\mathrm{CH}_{3}\right)$ & Antisymmetric stretching vibration \\
$2925 \pm 5 \mathrm{~cm}^{-1}$ & Methylene $\left(-\mathrm{CH}_{2}^{-}\right)$ & Antisymmetric stretching vibration \\
$2400 \sim 2300 \mathrm{~cm}^{-1}$ & Instrument background peak & - \\
$1900 \sim 1630 \mathrm{~cm}^{-1}$ & Carbonyl $(-\mathrm{C}=\mathrm{O})$ & Stretching vibration \\
$1620 \sim 1450 \mathrm{~cm}^{-1}$ & On benzene ring C=C & Stretching vibration \\
$1300 \sim 1000 \mathrm{~cm}^{-1}$ & Alcohol, phenol, ether $(\mathrm{C}-\mathrm{O})$ & Stretching vibration \\
\hline
\end{tabular}

2.2.2. Extraction of Fulvic Acid. A certain mass of samples were weighed from two test groups with 5\% sodium hydroxide (solid-liquid ratio 1:4) added and immersed for 24 hours. The $\mathrm{pH}$ was adjusted to $10 \sim 12$ after the distilled water of 20 times volume was added. Then, by heating at $80^{\circ} \mathrm{C}$ for 2 hours, the liquid supernatant was obtained through multiple centrifugation processing several times. The $\mathrm{pH}$ of liquid supernatant was adjusted to 3 by adding different liquid acids. The fulvic acid extracted from the methane fermentation group and acid precipitation by sulfuric acid, nitric acid, hydrochloric acid, and phosphoric acid were named FMS, FMN, FMH, and FMP, respectively. The fulvic acid was extracted from the unfermented group and acid precipitation by sulfuric acid (FS), nitric acid (FN), hydrochloric acid (FH), and phosphoric acid (FP), respectively (Table 1 below). And, all of the fulvic acid samples were taken and dried for testing.

2.2.3. Determination of Fulvic Acid Content. One milliliter of fulvic acid was diluted to $100 \mathrm{~mL}$ and filtered, $10 \mathrm{~mL}$ of the initial solution was discarded, then $5 \mathrm{~mL}$ of the filtrate was drawn into a $250 \mathrm{~mL}$ Erlenmeyer flask, $5.00 \mathrm{~mL}$ of $0.04 \mathrm{~mol} \cdot \mathrm{L}^{-1}$ potassium dichromate was added, and $16 \mathrm{~mL}$ of concentrated sulfuric acid was slowly added and then heated and oxidized in a boiling water bath for 30 minutes. After cooling to the room temperature, 3-5 drops of o-phenanthroline indicator was added, it was titrated to brown-red with calibrated ferrous ammonium sulfate, and then a blank test was made at the same time:

$$
B=\frac{0.003 \times\left(V_{0}-V_{1}\right) \times N}{C \times V},
$$

where $B$ is the content of fulvic acid, $\mathrm{mg} \cdot \mathrm{L}^{-1}, 0.003$ is the milligram equivalent of carbon, $\mathrm{mg}, V_{0}$ is the volume of ammonium ferrous sulfate consumed during titration blank, $\mathrm{L}, V_{1}$ is the volume of ammonium ferrous sulfate consumed during titration sample, $\mathrm{L}, N$ is the concentration of ferrous ammonium sulfate, $\mathrm{mol} \cdot \mathrm{L}^{-1}, C$ is the conversion factor for the humic acid carbon ratio (FAis the 0.54), and $V$ is the volume of the sample, $\mathrm{L}$.

\subsection{Spectral Analysis}

2.3.1. Fourier-Transform Infrared Spectroscopy. One milligram dried fulvic acid sample was taken and measured by FTIR spectroscopy, and it was recorded. The measurement conditions of all the samples were completely identical.
2.3.2. UV-Vis Spectroscopy. Ten milligram fulvic acid sample was weighed, which was dissolved with $70 \mathrm{~mL}$ of $0.05 \mathrm{~mol} \cdot \mathrm{L}^{-1}$ sodium bicarbonate solution. Then, the $\mathrm{pH}$ was adjusted to 8.0 with $1 \%$ sodium hydroxide or $0.1 \mathrm{~mol} \cdot \mathrm{L}^{-1}$ hydrochloric acid, and finally $0.05 \mathrm{~mol} \cdot \mathrm{L}^{-1}$ was used to make up to $100 \mathrm{~mL}$ with sodium bicarbonate. The absorption curve of all the samples was measured by the UV-visible near-infrared spectrophotometer, and the scanning wavelength range was $200 \mathrm{~nm}$ to $800 \mathrm{~nm}$. The absorbance was measured at $465 \mathrm{~nm}$ and $665 \mathrm{~nm}$, and the $E_{4} / E_{6}$ ratio was calculated.

2.3.3. Fluorescence Spectroscopy. Ten milligram fulvic acid sample was weighed, which was dissolved with $70 \mathrm{~mL}$ of $0.05 \mathrm{~mol} \cdot \mathrm{L}^{-1}$ sodium bicarbonate solution. Then, the $\mathrm{pH}$ was adjusted to 8.0 with $1 \%$ sodium hydroxide or $0.1 \mathrm{~mol} \cdot \mathrm{L}^{-1}$ hydrochloric acid, and finally $0.05 \mathrm{~mol} \cdot \mathrm{L}^{-1}$ was used to make up to $100 \mathrm{~mL}$ with sodium bicarbonate. And, the fluorescence spectra of all the samples were recorded on the fluorescence spectrophotometer. Scanning method: the scanning speed of both the emission and excitation monochromators was $1000 \mathrm{~nm} \cdot \mathrm{min}^{-1}$, and the slit width of both the emission and excitation monochromators was $8 \mathrm{~nm}$. The fluorescence emission spectrum was scanned from $275 \mathrm{~nm}$ to $650 \mathrm{~nm}$ with a fixed excitation wavelength of $274 \mathrm{~nm}$.

\section{Results and Discussion}

3.1. Yield of Fulvic Acid. The yield of fulvic acid significantly reduces after methane fermentation (Figure 1), the sulfuric acid precipitation group reduces from $5.33 \%$ (FS) to $4.19 \%$ (FMS), the nitric acid precipitation group reduces from $8.94 \%$ (FN) to $5.19 \%$ (FMN), the hydrochloric acid precipitation group reduces from $8.55 \%(\mathrm{FH})$ to $6.02 \%(\mathrm{FMH})$, and the phosphoric acid precipitation group reduces from $12.17 \%$ (FP) to $9.22 \%$ (FMP). It indicates that methane fermentation degrades and consumes part of the fulvic acid, resulting in a decrease in the yield of fulvic acid.

The yield of fulvic acid in the methane fermentation group and the unfermented group was the highest by phosphoric acid precipitation. The yield of FP was up to $12.17 \%$, while the yield of FMP was $9.22 \%$, indicating that the yield of fulvic acid is the highest between the methane fermentation group and the unfermented group by phosphoric acid in the process of acid precipitation. 

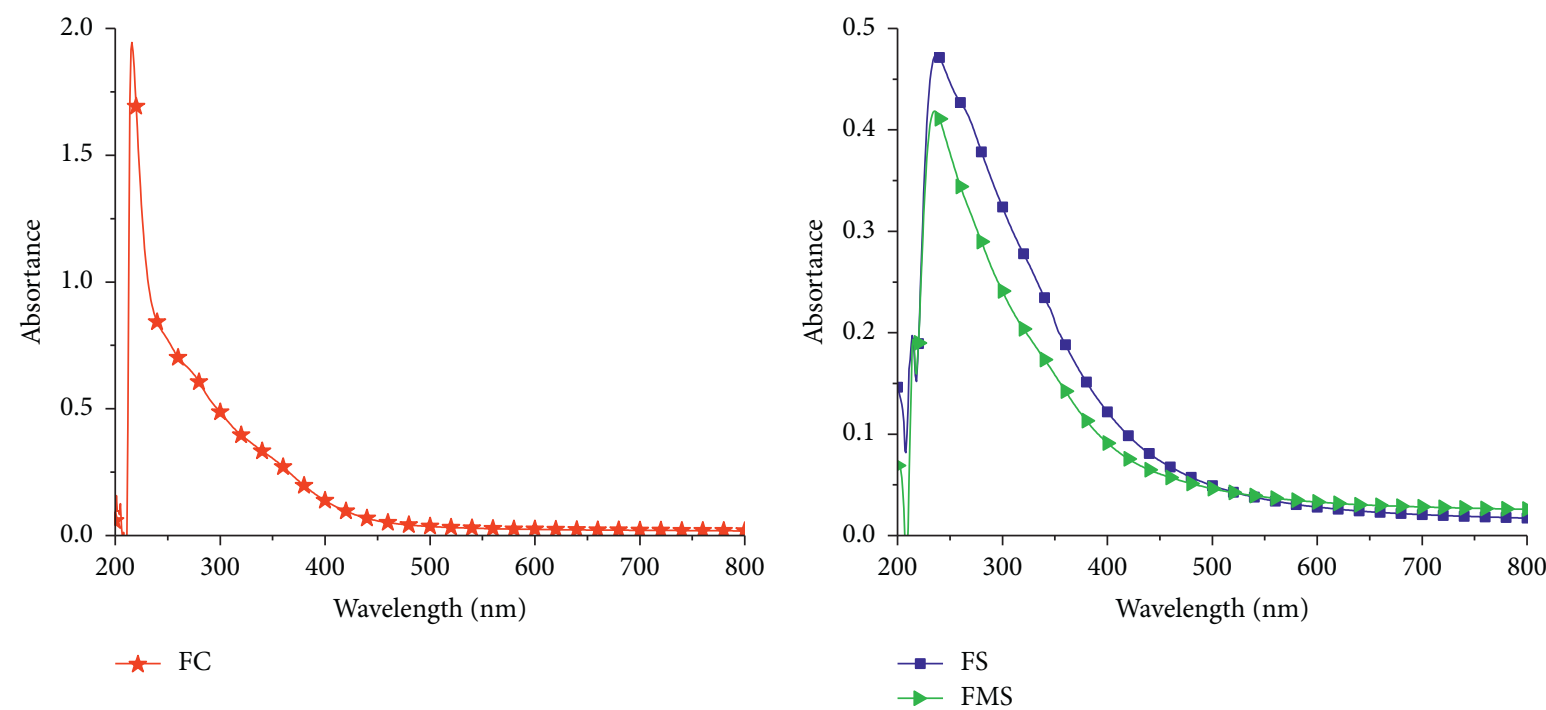

(a)

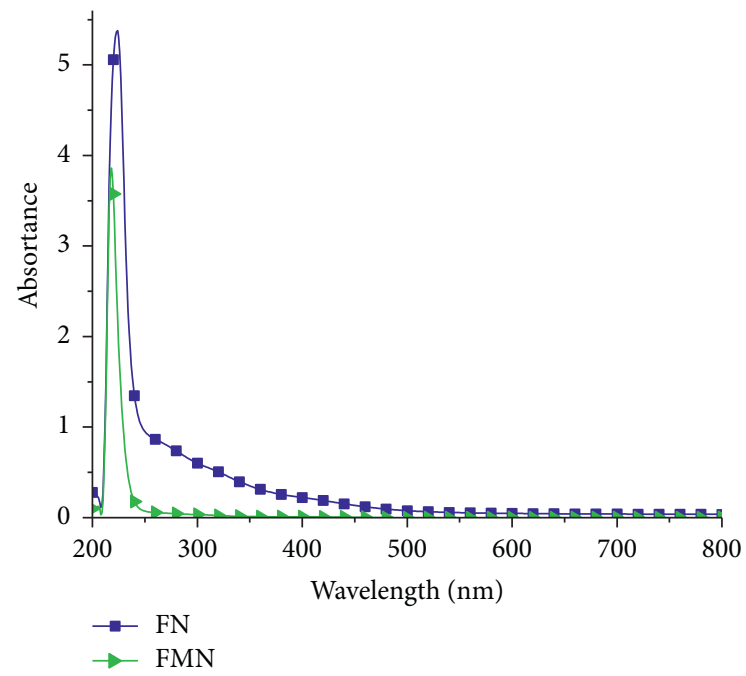

(c)

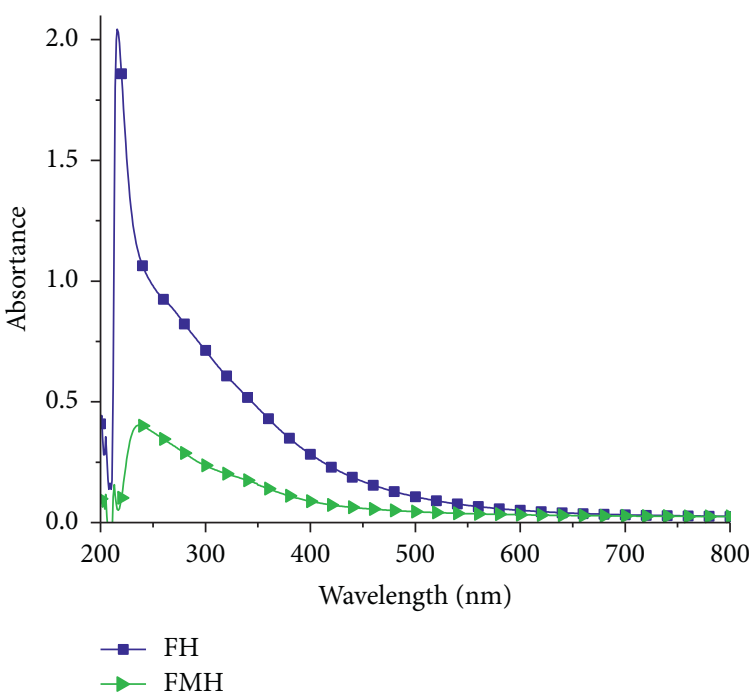

(d)

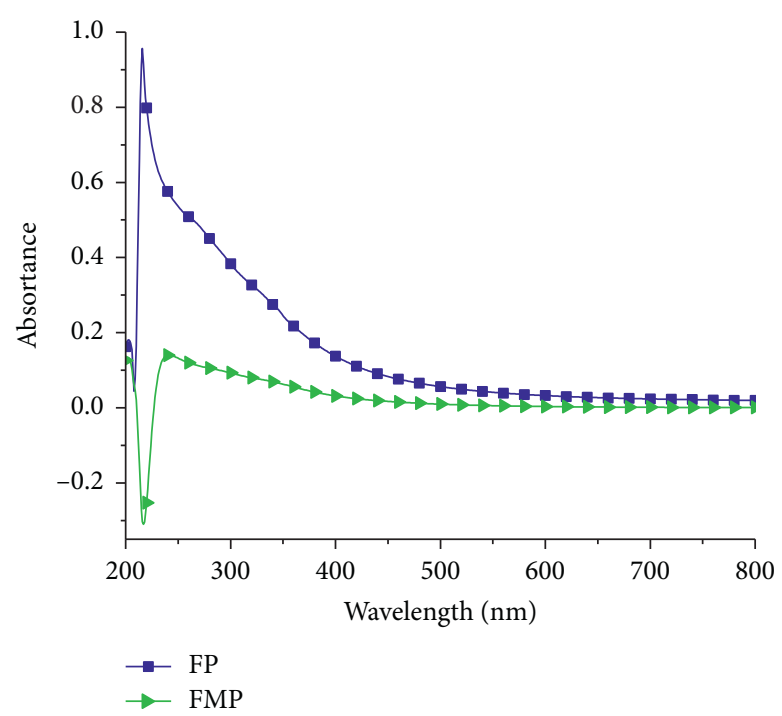

(e)

FIGURE 4: (a) UV-Vis of fulvic acid. UV-Vis of fulvic acid by (b) sulfuric acid precipitation, (c) nitric acid precipitation, (d) hydrochloric acid precipitation, and (e) phosphoric acid precipitation. 


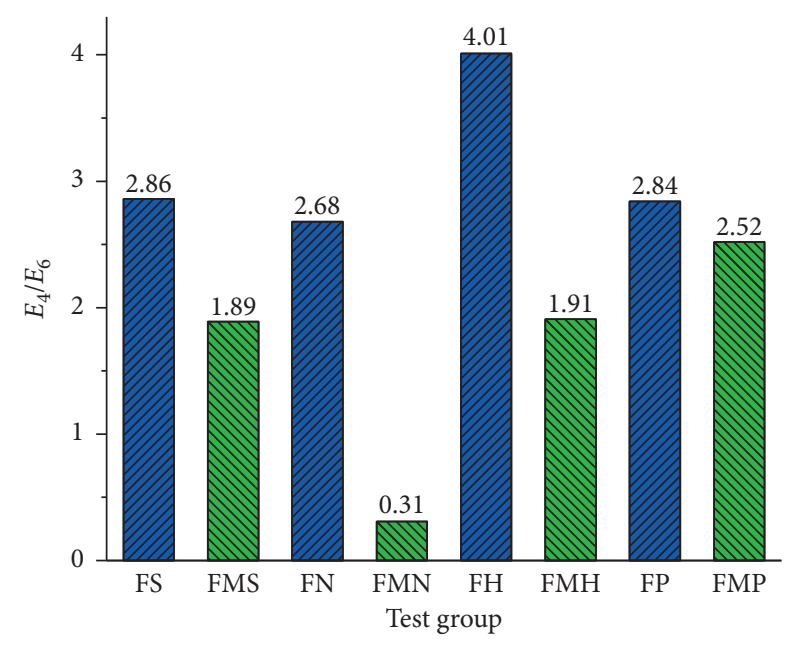

FIgURE 5: $E_{4} / E_{6}$ of fulvic acid.

3.2. Content of Fulvic Acid. The content refers to the proportion of fulvic acid of the supernatant liquid precipitated by different acids. The content of the fulvic acid obtained from the unfermented group is almost all higher than that of the fulvic acid extracted from the methane fermentation group (Figure 2). The sulfuric acid precipitation group decreases from $1.101 \mathrm{mg} \cdot \mathrm{L}^{-1}$ (FS) to $0.667 \mathrm{mg} \cdot \mathrm{L}^{-1}$ (FMS), the hydrochloric acid precipitation group decreases from $0.944 \mathrm{mg} \cdot \mathrm{L}^{-1}(\mathrm{FH})$ to $0.333 \mathrm{mg} \cdot \mathrm{L}^{-1}(\mathrm{FMH})$, and the phosphoric acid precipitation group decreases from $1.111 \mathrm{mg} \cdot \mathrm{L}^{-1}$ (FP) to $0.611 \mathrm{mg} \cdot \mathrm{L}^{-1}$ (FMP). It proves that the fulvic acid was degraded by microorganisms in the methane fermentation process, resulting in a decrease in the content of fulvic acid. The fulvic acid by nitric acid precipitation shows an anomaly, with the content of fulvic acid from the methane fermentation group being higher than that of the unfermented group, and it increases from $1.278 \mathrm{mg} \cdot \mathrm{L}^{-1}(\mathrm{FN})$ to $1.389 \mathrm{mg} \cdot \mathrm{L}^{-1}$ (FMN). It implies that the peat residue after methane fermentation was oxidized and modified the by nitric acid [10], leading to the increase of the fulvic acid content.

The highest content of fulvic acid is the precipitation by nitric acid, and the lowest content of fulvic acid is the precipitation by hydrochloric acid both in unfermented group and methane fermentation group. In addition, the content of FN and FMN is up to $1.278 \mathrm{mg} \cdot \mathrm{L}^{-1}$ and $1.389 \mathrm{mg} \cdot \mathrm{L}^{-1}$, respectively, and that of $\mathrm{FH}$ and $\mathrm{FMH}$ is only $0.944 \mathrm{mg} \cdot \mathrm{L}^{-1}$ and $0.333 \mathrm{mg} \cdot \mathrm{L}^{-1}$, respectively. It demonstrates that both in the unfermented group and the methane fermentation group, nitric acid is the best liquid acid to obtain the fulvic acid with high content in the process of extracting fulvic acid by the alkali solution acid precipitation method under different liquid acids.

3.3. Infrared Spectrum of Fulvic Acid. There are multiple absorption peaks in the mid-infrared range of $4000 \sim 400 \mathrm{~cm}^{-1}$ of fulvic acid extracted from the unfermented peat by acid precipitation with different liquid acids (Figure 3). The typical functional groups and the FTIR signal with the possible compounds are listed in Table 2 for reference [11-14]. FTIR of FC is shown in Figure 3(a) to provide a standard for other fulvic acid samples.

In the unfermented group, according to the peak intensity in $3700 \sim 3200 \mathrm{~cm}^{-1}$, the hydroxyl content is $\mathrm{FP}>\mathrm{FH}>\mathrm{FS}>\mathrm{FN}$ from high to low, with FP having a strong absorption peak at $3490 \mathrm{~cm}^{-1}$. FP has a strong absorption at $2970 \mathrm{~cm}^{-1}$, and $\mathrm{FH}$ is lower, indicating that methyl content of FP is the highest; FH is the second, and methyl content of FN and FS are similar. The absorption peak of methylene is at $2925 \pm 5 \mathrm{~cm}^{-1}, \mathrm{FP}$ and $\mathrm{FH}$ show a strong absorption peak at $2925 \mathrm{~cm}^{-1}$, the absorption peak of $\mathrm{FH}$ blue shifted to $2920 \mathrm{~cm}^{-1}$, the absorption peak of FP red shifted to $2928 \mathrm{~cm}^{-1}$, and the methylene content is $\mathrm{FP}>\mathrm{FH}>\mathrm{FS}>\mathrm{FN}$ from high to low. This is explained by the fact that a part of the weak covalent bonds in the aliphatic group of FN were broken with the oxidability of nitric acid, which generated oxygen-containing functional groups including phenolic hydroxyl, carbonyl, and carboxyl, resulting in a decrease of methyl and methylene groups. It is possible that all bands in the area from 1900 to $1630 \mathrm{~cm}^{-1}$ could be influenced by liquid acids, and the absorption peak intensity is $\mathrm{FP}>\mathrm{FN}>\mathrm{FH}>\mathrm{FS}$, with FN and FP containing more benzene rings. $\mathrm{FN}$ and $\mathrm{FP}$ show a sharp absorption peak at $1620 \sim 1450 \mathrm{~cm}^{-1}$, which proves that FN and FP contained more benzene rings. Additionally, the absorption band of $1300 \sim 1000 \mathrm{~cm}^{-1}$ shows a different pattern for FN compared with FS, FH, and FP, indicating that FN contained more hydroxyls or ether bonds than the others. FP contains more functional groups with the highest yield and the second highest content, indicating that phosphoric acid is the best liquid acid for the unfermented peat.

In the methane fermentation group, FMN has the strongest absorption at $3700 \sim 3200 \mathrm{~cm}^{-1}$, which shows that FMN includes the largest number of $-\mathrm{OH}$. Both in $2960 \pm 10 \mathrm{~cm}^{-1}$ and $2925 \pm 5 \mathrm{~cm}^{-1}$, FMP has the strongest absorption while FMN has the weakest absorption, so the content of methyl and methylene is FMP $>$ FMS $>$ FMH $>$ FMN from high to low. FMN sample characterizes by a broad strong absorption band at $1900 \sim 1630 \mathrm{~cm}^{-1}$, with moderate absorption bands around the $1600 \mathrm{~cm}^{-1}$ region. These results suggest that there are more carbonyls and benzene rings in FMN. In the range of $1300 \sim 1000 \mathrm{~cm}^{-1}$, FMN with more hydroxyl or ether bonds shows a strong absorption peak at $1046 \mathrm{~cm}^{-1}$. FMN had the highest content of various functional groups, so that nitric acid is the most appropriate liquid acid for the methane fermentation group.

After methane fermentation, the peak intensity of FMH and FMP decreases significantly, and the peak intensity of FMN reduces slightly. The peat residue was oxidized and modified by nitric acid in the methane fermentation process, enabling more fulvic acid to be precipitated [14]. In the range of $4000 \sim 1200 \mathrm{~cm}^{-1}$, FMS has the lower absorption peak after methane fermentation; while in the range of $1200 \sim 400 \mathrm{~cm}^{-1}$, the absorption peak of FMS increases. This may be caused by $\mathrm{H}^{+}$released by sulfuric acid during the acid precipitation process. The combination of metal ions and carboxyl groups of the fulvic acid would be broken by $\mathrm{H}^{+}$to form free fulvic 


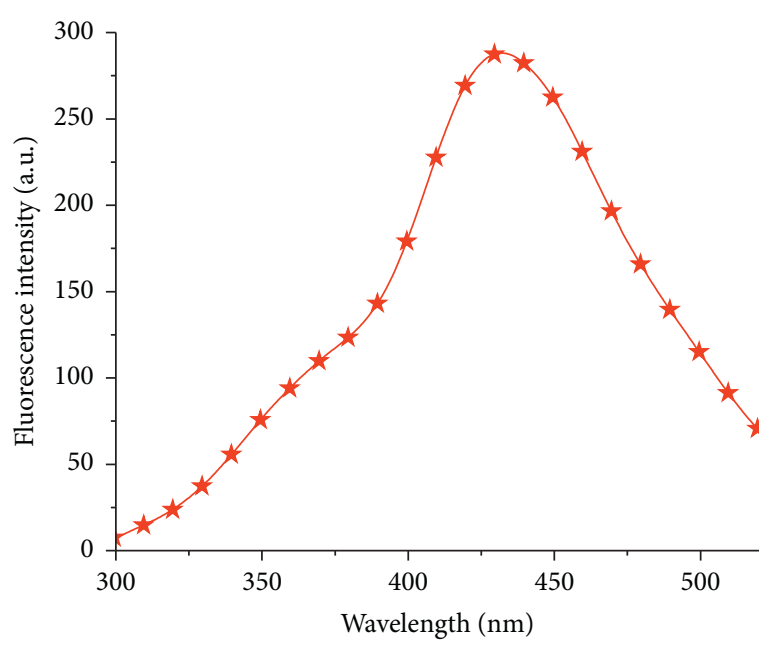

$\star$ FC

(a)

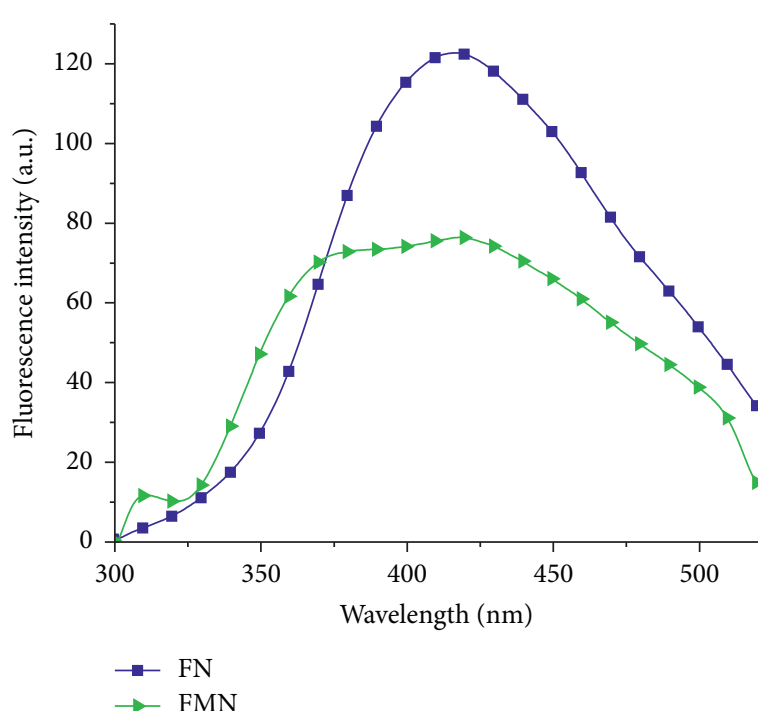

(c)

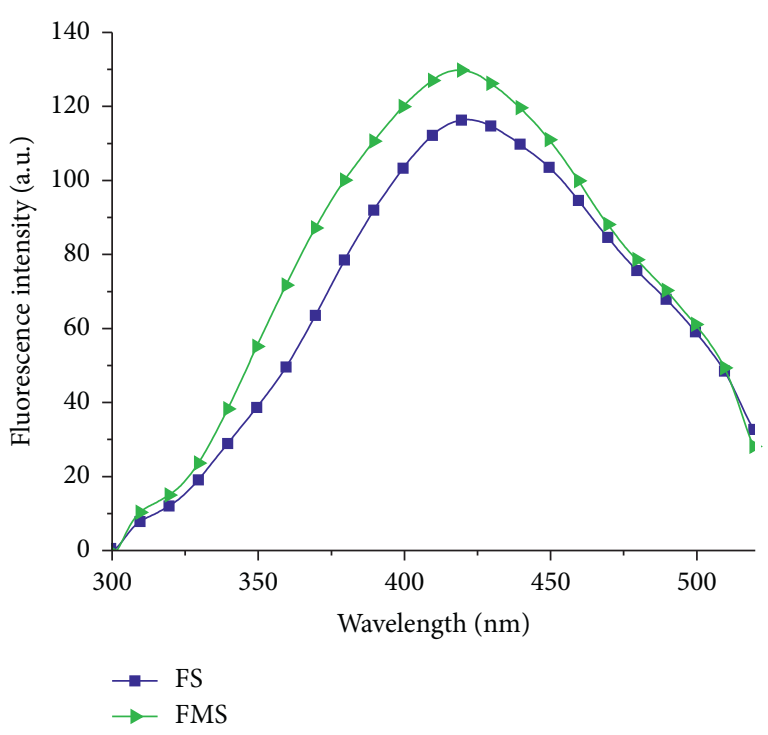

(b)

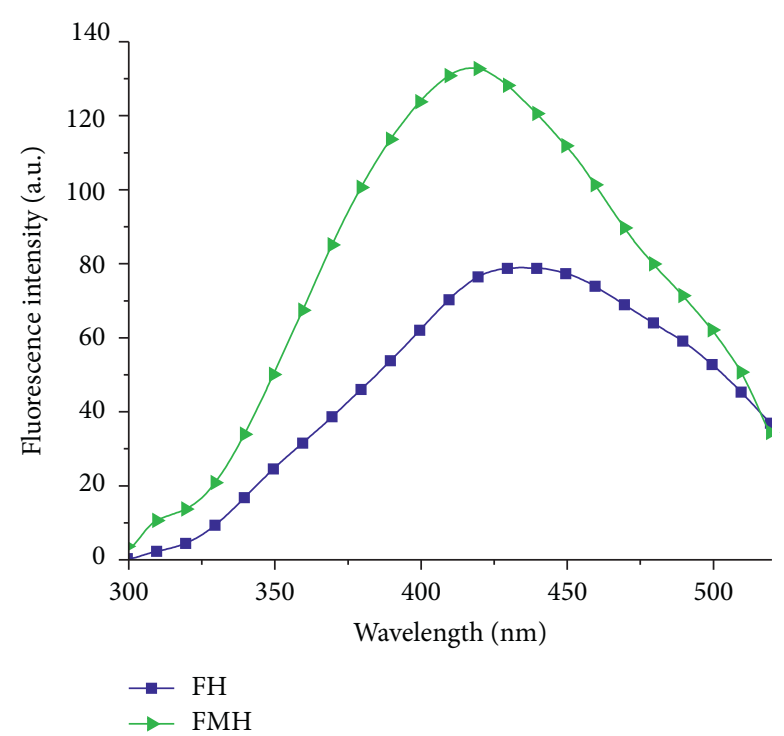

(d)

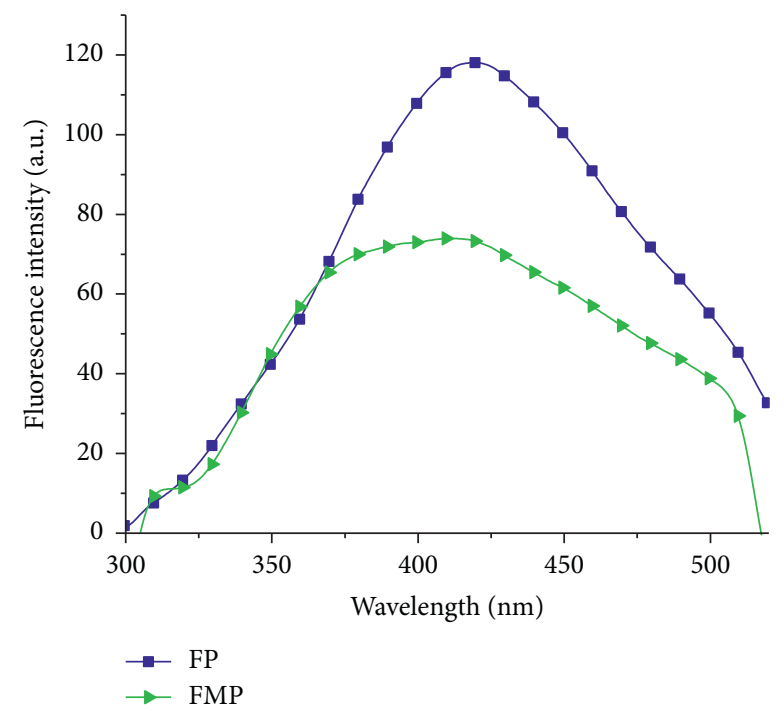

(e)

Figure 6: (a) Fluorescence spectrum of fulvic acid. Fluorescence spectrum of fulvic acid by (b) sulfuric acid precipitation, (c) nitric acid precipitation, (d) hydrochloric acid precipitation, and (e) phosphoric acid precipitation. 
acid. Extraction of fulvic acid requires the addition of more sulfuric acid to provide $\mathrm{H}^{+}$, resulting in the presence of more sulfate in the FMS and causing an increase in the ash of FMS [15]. During the methane fermentation process, some methyl groups were consumed, causing the $\mathrm{C}-\mathrm{O}$ bond of alcohols, phenols and ethers were broken which resulted both in the decrease of methyl, hydroxyl, and ether bonds and in the increase of methylene, carbonyl, and benzene rings.

3.4. UV-Vis Spectrum of Fulvic Acid. In the whole wavelength range of $200 \sim 800 \mathrm{~nm}$, a trend was shown that increases first and then decreases in Figure 4, which might be attributed to the diversity of the chromophore group and extended conjugation [16]. All of the samples have a strong UV-visible absorption existing in the near ultraviolet range $(200 \sim 250 \mathrm{~nm})$. The maximum absorption wavelength $\left(\lambda_{\max }\right)$ of FC appeared at $213 \mathrm{~nm}$ (Figure 4(a)). This is the $\pi \longrightarrow \pi^{*}$ transition of the characteristic absorption band ( $\mathrm{E}$ band) of the aromatic benzene ring conjugated double bond. The $\lambda_{\max }$ of FMH red shifted from $216 \mathrm{~nm}(\mathrm{FH})$ to $237 \mathrm{~nm}$, and the $\lambda_{\text {max }}$ of FMP also red shifted from $214 \mathrm{~nm}$ (FP) to $241 \mathrm{~nm}$ (Figures 4(d) and 4(e)). However, the blue shift of fulvic acids precipitated by sulfuric acid and nitric acid is observed in Figures 4(b) and 4(c); it shows that the utilization of liquid acids made a significant influence on the structure of fulvic acid.

In the hydrochloric acid group and phosphoric acid group (Figures $4(\mathrm{~d})$ and $4(\mathrm{e})$ ), the $\lambda_{\max }$ shifts towards the longer wavelength after methane fermentation; it is found that the fulvic acid samples are a kind of condensed aromatic, with both the number of rings and the conjugated bond increasing. The structure of the benzene ring contains chromophore including phenolic hydroxyl groups, benzene carboxylic groups, and conjugated double bonds, resulting in a $\pi \longrightarrow \pi^{*}$ transition and $\pi \longrightarrow \pi^{*}$ transition occurring together. A blue shift was found in the sulfuric acid group and nitric acid group (Figures 4(b) and 4(c)) after methane fermentation; it can be speculated that the FMS may contain more sulfonic group $\left(-\mathrm{SO}_{3} \mathrm{H}\right)$ and $\mathrm{FMN}$ may contain many nitro groups $\left(-\mathrm{NO}_{2}\right)$, which causes the $\lambda_{\max }$ of FMS and FMN to move to the shorter wavelength.

The $E_{4} / E_{6}$ ratio is defined as the ratio of absorbance at wavelengths of $465 \mathrm{~nm}$ and $665 \mathrm{~nm}$. It is one of the important indicators for characterizing the composition structure of humic acids. Most scholars believe that it relates to the degree of aromatization or conjugate [17]. The $E_{4} / E_{6}$ ratio is inversely proportional to the degree of aromatization or conjugate, that is, the larger the $E_{4} / E_{6}$ ratio, the lower the molecular complexity, and the lower the degree of aromatization or conjugate. The $E_{4} / E_{6}$ ratio decreases after methane fermentation (Figure 5), demonstrating that the aromatization of fulvic acid samples in the methane fermentation group is higher than that of fulvic acid samples in the unfermented group. The methane fermentation would consume the groups of fulvic acid with simple structure including aliphatic chain hydrocarbons, while the composition structure with higher aromatization and conjugate cannot be consumed.
The $E_{4} / E_{6}$ ratio of $\mathrm{FH}$ in the unfermented group and FMP in the methane fermentation group are the highest with the simplest molecular structure. The $E_{4} / E_{6}$ ratio of $\mathrm{FN}$ and FMN is the lowest, which indicates that the fulvic acid by nitric acid precipitation has a higher degree of aromatization.

3.5. Fluorescence Spectrum of Fulvic Acid. The fluorescence spectrum of fulvic acid shows a trend of increasing first and then decreasing with wavelength increase, and the broad absorption peaks appear at the wavelength of $450 \mathrm{~nm}$ (Figure 6). The maximum fluorescence absorption intensity of fulvic acid extracted from the methane fermentation group is lower than that of fulvic acid in the unfermented group. This is because the fulvic acid obtained from the methane fermentation group contains more complicated structures, a higher degree of aromatization including carbonyl group and benzene rings, and the fluorescence is quenched due to the influence of solvent and temperature of solution.

The structural complexity of the fulvic acid obtained by different liquid acid precipitation is different. The maximum fluorescence peak of FH and FP is observed at $417 \mathrm{~nm}$ and $425 \mathrm{~nm}$, blue shifted to $415 \mathrm{~nm}$ (FMH) and $416 \mathrm{~nm}$ (FMP) after methane fermentation, respectively. The blue-shifted fluorescence peaks are believed to be due to the simple structure including aliphatic chain hydrocarbons of FMH and FMP utilized by the microorganisms during the methane fermentation process, resulting in that FMH and FMP had high degree of aromatization and complex structure (Figures 6(d) and 6(e)). A clear red shift occurs in the sulfuric acid group and nitric acid group (FS $\longrightarrow$ FMS: $418 \mathrm{~nm} \longrightarrow 420 \mathrm{~nm}, \quad \mathrm{FN} \longrightarrow$ FMN: $414 \mathrm{~nm} \longrightarrow 417 \mathrm{~nm}$.) (Figures 6(b) and 6(c)). As sulfuric acid has strong acidity and nitric acid has strong oxidizing nature, an abnormal phenomenon occurred in FMS and FMN $[14,15]$.

\section{Conclusion}

(1) The yield and content of fulvic acid extracted from the peat residue after methane fermentation significantly reduces, and it shows that part of fulvic acid will be degraded during methane fermentation. With different liquid acid precipitation, the yield of fulvic acid by phosphoric acid precipitation is the highest and the highest content of fulvic acid is by nitric acid precipitation.

(2) FP is the best choice for acid precipitation of unfermented peat with the largest number of various functional groups. FMN contains the largest number of various functional groups, so nitric acid is the better choice for peat residue after methane fermentation. Methane fermentation leads to the increase of methylene, carbonyl, benzene ring, and other functional groups and consumes simple and easy-to-use groups in fulvic acid, resulting in the reduction of methyl and hydroxyl groups. 
(3) The UV-visible spectrum of fulvic acid shows that it makes a significant influence on the structure of fulvic acid by different liquid acid precipitation. FMS contains more $-\mathrm{SO}_{3} \mathrm{H}$, and $\mathrm{FMN}$ contains more $-\mathrm{NO}_{2}$. The $E_{4} / E_{6}$ ratio of fulvic acid decreases after methane fermentation; the simpler structure of fulvic acid is consumed and utilized during the methane fermentation process while the structure with a higher degree of aromatization and conjugate cannot be consumed. The fulvic acid with simplest molecular structure by hydrochloric acid precipitation can be used by microorganisms easily.

(4) The fluorescence spectrum of fulvic acid finds that the methane fermentation group contains more complicated structures and a higher degree of aromatization. As sulfuric acid has strong acidity and nitric acid has strong oxidizing nature, FMS and FMN display some anomalous phenomenon in spectrum analysis.

\section{Data Availability}

The data(opj) used to support the findings of this study are included within the supplementary information files.

\section{Conflicts of Interest}

The authors declare that there are no conflicts of interest regarding the publication of this paper.

\section{Acknowledgments}

High tribute shall be paid to Mr. Lee A. LIBBEY and Mrs. Xiuli SHI, who had offered me valuable suggestions for this article. They spent much time to read and point out the errors. The completion of this thesis would not have been possible without their help. The authors are grateful to the financial support provided by the National Natural Science Foundation of China (No. 21766025), Natural Science Foundation of Inner Mongolia (No. 2017MS(LH)0210), Scientific Research Project of Universities in Inner Mongolia Autonomous Region (No. NJZY17166), and Ministry of Education "Chun Hui Plan" Cooperative Research Project (No. 2018042).

\section{Supplementary Materials}

Date 1: yield of fulvic acid. Date 2: content of fulvic acid. Date 3: FTIR of fulvic acid. Date 4: UV-Vis of fulvic acid. Date 5: E4 E6 of fulvic acid. Date 6: fluorescence spectrum of fulvic acid. (Supplementary Materials)

\section{References}

[1] E. V. Lazareva, I. N. Myagkaya, I. S. Kirichenko, M. A. Gustaytis, and S. M. Zhmodik, "Interaction of natural organic matter with acid mine drainage: in-situ accumulation of elements," Science of The Total Environment, vol. 660, no. 10, pp. 468-483, 2019.
[2] T. Jia, X. Yukun, and L. Yaning, "Agricultural and forestry waste composts as substitutes for peat in potting media: effects on root growth and fractal features of new guinea impatiens (Impatiens hawkeri)," Agricultural Research, vol. 5, no. 3, pp. 269-276, 2016.

[3] M. Papaioanou, E. G. Chronopoulou, G. Ciobotari et al., "Evaluation of the nutraceutical and cosmeceutical potential of two cultivars of Rubus fruticosus L. under different cultivation conditions," Current Pharmaceutical Biotechnology, vol. 18, no. 10, pp. 890-899, 2017.

[4] V. Antoniadis, A. Zanni, E. Levizou et al., "Modulation of hexavalent chromium toxicity on Origanum vulgare in an acidic soil amended with peat, lime, and zeolite," Chemosphere, vol. 195, pp. 291-300, 2018.

[5] W. Wei, W. Jinggui, L. Yunhui et al., "Effects of organic materials on the composition and structure of humic substance in the rhizosphere soil of different crops," Journal of Soil \& Water Conservation, vol. 31, no. 2, pp. 215-220, 2017.

[6] M. Yunfei, Y. Qisheng, Z. Yanling et al., "Composition characters of soil humus and their relationships with routine chemical components in tobacco leaves in middle henan tobacco growing areas," Tobacco Science \& Technology, vol. 50, no. 4, pp. 31-36, 2017.

[7] W. Ding, J. Cui, Y. Zhao et al., "Enhancing Haematococcus pluvialis biomass and $\gamma$-aminobutyric acid accumulation by two-step cultivation and salt supplementation," Bioresource Technology, vol. 285, Article ID 121334, 2019.

[8] E. S. Trofimova, M. V. Zykova, A. A. Ligacheva et al., "Influence of humic acids extracted from peat by different methods on functional activity of macrophages in vitro," Bulletin of Experimental Biology and Medicine, vol. 162, no. 6, pp. 741-745, 2017.

[9] Z. Yuanping, C. Gang, Z. Guolan et al., "Study on extraction of biological humic acids from fermented furfural residue," Agricultural Science \& Technology, vol. 17, no. 6, pp. 1442$1445,2016$.

[10] K. A. Thorn, L. G. Cox, and M. Jingdong, "Nitrosation and nitration of fulvic acid, peat and coal with nitric acid," PLoS One, vol. 11, no. 5, Article ID e0154981, 2016.

[11] M. Tatzber, M. Stemmer, H. Spiegel et al., "FTIR-spectroscopic characterization of humic acids and humin fractions obtained by advanced $\mathrm{NaOH}, \mathrm{Na}_{4} \mathrm{P}_{2} \mathrm{O}_{7}$, and $\mathrm{Na}_{2} \mathrm{CO}_{3}$ extraction procedures," Journal of Plant Nutrition and Soil Science, vol. 170, no. 4, pp. 522-529, 2007.

[12] Z. Song, K. W. Jones, N. Marinkovic, X. M. Xiao, H. Feng, and E. Tchouparova, "Characterization of organic contaminants in New York/New Jersey harbor sediments using FTIR-ATR and synchrotron FTIR," CLEAN-Soil, Air, Water, vol. 39, no. 12, pp. 1041-1049, 2011.

[13] L. Yonghong and S. Pohsin, "Behavior of aluminum adsorption in different compost-derived humic acids," CLEANSoil, Air, Water, vol. 38, no. 10, pp. 916-920, 2010.

[14] G. Yuxuan and C. Shushi, "Fluorescence quenching study of humic-fraction-modified silica gel in a solid state after association with a variety of pesticidal analytes in hexane and acetonitrile," Journal of the Chinese Chemical Society, vol. 65, no. 8, pp. 995-1006, 2018.

[15] M. Eshwar, M. Srilatha, K. B. Rekha, and S. H. K. Sharma, "Complexation behavior of humic and fulvic acids with metal ions and their assessment by stability constants," International Journal of Pure \& Applied Bioscience, vol. 5, no. 6, pp. 899-907, 2017.

[16] M. S. Twardowski, E. Boss, J. M. Sullivan, and P. L. Donaghay, "Modeling the spectral shape of absorbing chromophoric 
dissolved organic matter," Marine Chemistry, vol. 89, no. 1-4, pp. 69-88, 2004.

[17] P. Zalba, N. M. Amiotti, J. A. Galantini, and S. Pistola, "Soil humic and fulvic acids from different land use systems evaluated by $E_{4} / E_{6}$ ratios," Communications in Soil Science and Plant Analysis, vol. 47, no. 13-14, pp. 1675-1679, 2016. 